Dossier

DOI: $10.26807 /$ cav.vi10.339

\title{
LA INVESTIGACIÓN-CREACIÓN COMO PRODUCCIÓN DE NUEVO CONOCIMIENTO: PERSPECTIVAS, DEBATES Y DEFINICIONES
}

\section{RESEARCH-CREATION AS PRODUCTION OF NEW KNOWLEDGE: PERSPECTIVES, DEBATES AND DEFINITIONS}

Elsa María Beltrán-Luengas

Alejandro Villaneda Vásquez

\section{Resumen}

Aunque se han hecho grandes avances en el reconocimiento de los productos de la investigación-creación en el sistema nacional que regula la investigación en Colombia, persisten resistencias a la hora de pensar que el arte puede producir nuevo conocimiento. En efecto, existen dificultades en pensar que este puede inscribirse en artefactos plástico-sensoriales, propios de la creación artística. De hecho, los creadores parecen no tener las suficientes herramientas argumentativas para demostrar que sus procesos creativos pueden producir nuevos conocimientos y así, acceder a los recursos de investigación y a las clasificaciones como investigadores. En este artículo proponemos una definición del concepto de investigacióncreación en términos de su resultado, de las características de los conocimientos que produce y de sus fases, y sugerimos cuál es la función del creador en los procesos inter y transdisciplinarios. Concluimos con un énfasis en la diferencia del conocimiento que produce el arte con relación al que produce la ciencia. 
Palabras clave: Artefacto; disciplinas creativas; investigación-creación; nuevo conocimiento; plástico-sensorial; transdisciplinar.

\begin{abstract}
Although great advances have been made in recognizing the products of research-creation in the national research system in Colombia, resistance persists when thinking that art can produce new knowledge. Indeed, there are still difficulties in thinking that new knowledge may be inscribed in plastic-sensory artifacts, typical of artistic creation. In fact, it seems to be that creators don't have the argumentative tools to demonstrate that their creative processes can produce new knowledge and thus, getting access to research resources, as well as being classified as researchers. In this article we propose a definition of the concept of research-creation in terms of its result, the features of the knowledge it produces, as well as its phases; also, we suggest the role of the creator in inter and transdisciplinary processes. We conclude with an emphasis on the difference between the knowledge produced by art and the one produced by science.
\end{abstract}

Keywords: Artifact; creative disciplines; research-creation; new knowledge; plastic-sensory; transdisciplinary.

\title{
Biografía de los autores
}

Elsa María Beltrán-Luengas (Bogotá, Colombia, 1976). Antropóloga, magíster en Antropología y candidata a Doctora en Bioética. Profesora asociada de la Facultad de Creación y Comunicación de la Universidad El Bosque (Bogotá). Se ha desempeñado en diversas áreas del conocimiento, cuyo punto de encuentro es la reflexión del aporte de las metodologías cualitativas en la producción de conocimientos situados. Entre sus últimas publicaciones se encuentran el artículo Las vicisitudes de la investigación artística y ambiental en los comités de ética (2019) publicado en la Revista Colombiana de Bioética y el libro ¿Investigar creando?: una guía para la investigación-creación en la academia (2018) de la editorial Universidad El Bosque. 
Código de identificación ORCID: http://orcid.org/0000-0001-7890-9909

Alejandro Villaneda Vásquez (Bogotá, Colombia, 1976). Diseñador industrial, especialista en Docencia Universitaria, con estudios en Filosofía de la Ciencia y estudiante del Doctorado en Diseño, Fabricación y Gestión de Proyectos Industriales de la Universidad Politécnico de Valencia. Profesor asociado de la Facultad de Creación y Comunicación de la Universidad El Bosque desde el año 2002. Tiene amplia experiencia en diseño instruccional y en la formación por competencias, así como en la dirección y en el desarrollo de proyectos de investigación en torno a la valorización del territorio entre los que se destacan Los secretos del Venado Dorado y Mundos invisibles. Énfasis en diseño experiencial y de emociones.

Código de identificación ORCID: http://orcid.org/0000-0002-8688-1077

\section{Introducción}

Recientemente, el Sistema Nacional de Ciencia, Tecnología e Innovación de Colombia reconoció como productos de investigación los resultados de algunos procesos de creación e investigación-creación. Este reconocimiento fue el resultado de la gestión de la Mesa de Artes, Arquitectura y Diseño (AAD) junto con el entonces Departamento Administrativo de Ciencia, Tecnología e Innovación (Colciencias) y actualmente Ministerio de Ciencia, Tecnología e Innovación (Minciencias). La Mesa de ADD se constituyó, en el año 2013, como un grupo de

profesores universitarios pertenecientes a la Asociación Colombiana de Facultades y Programas de Artes (ACOFARTES), la Asociación Colombiana de Facultades de Arquitectura (ACFA) y la Red Académica de Diseño (RAD Colombia), quienes pusieron sobre la mesa la discusión acerca de la posibilidad de producir nuevo conocimiento en estas áreas y de cómo medir estos procesos en el marco de un sistema nacional de investigación.

Una de las razones más importantes para avanzar en este debate tuvo que ver con que la producción de conocimiento que se inscribía en obras o productos artísticos en el ámbito académico no se estaba reconociendo, lo que resultaba en una desventaja de los creadores frente a los investigadores de otros saberes, dado que no podían acceder a clasificaciones altas; en ese 
sentido, tendían a desviar su producción hacia los productos como artículos y libros de investigación, que correspondían a aquellos con mayor peso en el modelo de medición ( $\mathrm{T}$. Delgado $^{1}$, comunicación personal, noviembre de 2015).

En 2015, Colciencias invitó a participar en su convocatoria 737 a todos los grupos de investigación del país que quisieran ser reconocidos y clasificados y, por primera vez, se realizó una medición de los productos de la creación en Artes, Arquitectura y Diseño. Los resultados fueron notables: los productos de estas áreas aumentaron en un 766\% con relación a la medición anterior (Departamento Administrativo de Ciencia, Tecnología e Innovación [Colciencias], 2016).

A partir de entonces, los investigadores de áreas creativas han tenido la posibilidad de escalar en la pirámide de clasificación. No obstante, aún hace falta consolidar la argumentación sobre cómo se puede producir nuevo conocimiento en el arte, en el diseño y en la arquitectura. Hoy por hoy, al menos en la sociedad colombiana, se tiende a relacionar la investigación únicamente con las ciencias naturales enmarcadas en parámetros de generalización y reproducibilidad de los resultados. En ese sentido, se convierte en prioridad que profesores y estudiantes de disciplinas artísticas conozcan cómo pueden discutir y tomar posición acerca de la producción de nuevo conocimiento en el arte y sobre cómo este se relaciona con otros campos de saber, a fin de que puedan desempeñarse en contextos multi, inter y transdisciplinarios y valorar los posibles aportes que pueden hacer a la sociedad desde su disciplina, así como acceder a recursos para la financiación de sus proyectos.

Este artículo, tiene como objetivo, entonces, proponer una definición del concepto de investigación-creación y argumentar cómo se hace posible la inscripción de nuevo conocimiento en artefactos plástico-sensoriales, productos de las disciplinas artísticas. En primer lugar, propondremos, siguiendo a otros autores, los diferentes tipos de investigación que se puede realizar en los ámbitos creativos. Segundo, sugerimos una definición de investigación-

\footnotetext{
${ }^{1}$ Tania Delgado, miembro de la Mesa de ADD de 2013 a 2017 y última Directora de Mentalidad y Cultura de Minciencias-Colombia.
} 
creación con relación a la cual iremos explicando cada uno de sus componentes. Finalmente, describiremos las distintas fases de la investigación-creación, a fin de que pueda establecerse una estructura base para la formulación o descripción de proyectos de esta índole.

\section{Las diferentes aproximaciones a la investigación en disciplinas artísticas}

El debate sobre la relación entre la investigación y la creación ha sido bastante confuso debido a las llamadas “tricotomías preposicionales” (Ballesteros y Beltrán, 2018, p. 32), que consisten en unir la palabra investigación con la palabra creación mediante una preposición, así: investigación para la creación, investigación dentro de la creación, investigación sobre o acerca de la creación, investigación a través de la creación, investigación en la creación, investigación desde la creación, etc. Para aumentar la confusión, se introdujo el término investigación-creación, que aparece con guion (investigación-creación), con el símbolo más (investigación + creación), con espacio (investigación creación) o con el vocablo y (investigación y creación). A partir la síntesis ofrecida por Ballesteros y Beltrán (2018) construimos una forma que permite identificar el tipo de proyecto que se quiere formular o describir siguiendo la propuesta de las perspectivas instrumental, interpretativa y performativa propuesta por Borgdorff (2007).

En ese orden de ideas, de acuerdo con este autor, la perspectiva instrumental consiste en la formulación de proyectos que busquen desarrollar alguna herramienta, sistema, modelo o tecnología que faciliten o aporten al proceso creativo. Autores como Frayling (1993), Findeli (1998) y Asprilla (2014) reconocen esta perspectiva bajo la expresión investigación para la creación, y aunque existen sutiles diferencias entre ellos, están de acuerdo con que esta corresponde a estudios que se ponen al servicio de la práctica artística y entrega conocimiento acerca de los materiales, tecnologías, técnicas o métodos, entre otros, que se necesitan para el proceso creativo. Es posible que aquellos que quieran enmarcarse en este tipo de proyectos necesiten articularse con disciplinas como la ingeniería, la psicología, la administración, así como la logística y la sociología como lo vemos en el ejemplo a continuación.

Un caso interesante de la perspectiva instrumental es el del $N C L A B$, un laboratorio de pensamiento creativo desarrollado por el grupo NC-arte orientado a la democratización del arte. Dicho laboratorio se creó en 2014 en Bogotá (Colombia) y, cada dos años, durante cuatro 
jornadas, desarrolla una serie de talleres en los que artistas y espectadores provenientes de contextos diversos se encuentran para llevar a cabo procesos de creación experimental y colaborativa (NC-Arte, 2015). De este modo, el resultado de su proyecto es el taller y el proceso de apropiación de las artes por parte de los participantes, y no las obras resultantes del mismo.

La perspectiva interpretativa se refiere al estudio de la creación en términos de sus contextos, técnicas y contenidos y es llevada a cabo, con frecuencia, en articulación con disciplinas como la historia, la filosofía o la antropología. Autores como Findeli, Brouillet, Martin, Moineau y Tarrago (2008) y Londoño (2013) explican que esta perspectiva puede desarrollarse, también, por disciplinas diferentes a las creativas dado que su interés es la interpretación de la creación y de los procesos creativos de acuerdo con un contexto político, económico, histórico, geográfico, cultural, social o estético. En ese orden de ideas, este tipo de investigación se tiende a realizar a través de metodologías de corte cualitativo que informen sobre los modos de apropiación de la creación en un contexto determinado.

Para ilustrar la perspectiva interpretativa, podemos mencionar el proyecto Cuerpo femenino y abyección en los desnudos femeninos de Débora Arango propuesto por la politóloga y filósofa Beira Aguilar y el abogado Iván Mahecha (2012). Estos autores analizan el carácter transgresor de la obra de la artista colombiana Débora Arango desde tres ángulos: primero, desde la definición del concepto de abyección; segundo, desde el análisis de la obra a partir de lo estético y de lo conceptual; para finalizar con una propuesta de triangulación de los dos primeros ángulos, sugiriendo como evidencias las emociones y comentarios suscitados en la época en la que comenzó a circular la obra de Arango.

Finalmente, la perspectiva performativa la entendemos como el proceso de investigacióncreación; es decir, se da a partir de un proceso creativo que da como resultado una creación en la que se inscribe un nuevo conocimiento en términos de los elementos perceptuales y sensoriales. De acuerdo con Borgdorff (2007), esta perspectiva es la más controvertida y la llama también perspectiva de la acción, perspectiva inmanente o investigación en las artes. Niedderer (2013) sostiene que la dificultad de comprender esta perspectiva radica en que la 
investigación y la práctica se ha separado conceptualmente, por lo cual el conocimiento que se deriva directamente de los procesos creativos se suele calificar como "tácito" y se caracteriza por ser fuertemente experiencial y procedimental por lo que se construye sobre, lo que podríamos llamar, datos eminentemente sensoriales.

Mika Hannula, Juha Suoranta y Tere Vaden (2005) coinciden con Borgdorff (2007) en que la característica prominente de esta perspectiva es que no separa el sujeto del objeto o, en otras palabras, al creador de la creación o del contexto. En ese sentido, estas autoras reconocen y utilizan en su práctica creativa métodos diversos que procuran no caer en este tipo de dualismos y que reconocen los procesos intersubjetivos como parte fundamental del proceso de investigación, tales como la conversación y el diálogo, los casos de estudio colaborativos y la etnografía contemporánea. Así, la investigación artística sucede en una alternancia de elementos conceptuales y materiales que ocurre de forma iterativa y, de alguna manera, anárquica, en el sentido que expuso Feyerabend (1976) hace ya varias décadas: la complejidad que implica la producción de conocimiento no puede reducirse a unas pocas reglas, por lo que la transgresión hace parte esencial de la producción de nuevo conocimiento.

El caso de la obra Fragmentos de la artista colombiana Doris Salcedo (2018) es un caso que puede ilustrar la investigación performativa o investigación-creación. La obra en mención es un contra-monumento elaborado tras la firma de los acuerdos de paz del gobierno colombiano con la guerrilla de las Fuerzas Armadas Revolucionarias de Colombia (FARC) y que pretende, según Juliana Martínez, "representar, cuestionar y reflexionar sobre los conflictos y la sociedad en un momento determinado" (2020, p. 43); es decir, sobre cómo impacta la entrega de las armas por parte de las FARC, después de más de cincuenta años de conflicto interno, desde el significado mismo del acto. Esta obra consiste en la intervención de una casa de tipo colonial, cercana a los centros de poder en Bogotá, en cuyo piso se instalaron láminas de metal obtenidas a partir de la fundición de las armas entregadas y de su martillado por parte de mujeres víctimas del conflicto armado. En este ejemplo, vemos cómo un significado particular — como lo 
pueden ser el final del conflicto armado, el reconocimiento de las víctimas y el inicio de una época de paz en Colombia, por ejemplo— se inscribe en la materialidad de la obra.

\section{Propuesta de definición: ¿qué es la investigación-creación?}

De acuerdo con lo que uno de nosotros ha propuesto en otros escenarios, entendemos la investigación creación como "la producción de nuevos conocimientos que se inscriben necesariamente en un artefacto plástico-sensorial que aporte de manera significativa a las disciplinas creativas o a los contextos dentro de los que aquellos se insertan." (Beltrán, en prensa). Esta propuesta de definición tiene diferentes componentes complejos, de manera que iremos explicando uno a uno.

En primer lugar, entendemos la producción de nuevos conocimientos como contribuciones significativas desde y para las áreas creativas y en articulación con otras disciplinas, a fin de mejorar la experiencia del ser humano en un contexto dado. Para evitar caer en ambigüedades o imprecisiones (Hatch, 2019), seguimos la propuesta de Findeli y Coste (2007) para aclarar lo que entendemos por contribuciones significativas. De acuerdo con estos autores, los aportes en las áreas creativas deben cumplir las siguientes condiciones: primero, deben anclarse a la formulación y ejecución de un proyecto y, por lo tanto, a una situación singular con relación a la cual se quiere modificar el curso de las cosas. Segundo, dicho proyecto debe ser contemplado desde un punto de vista empírico, es decir, debe permitir la observación del mundo exterior del mismo (presupuesto y financiadores, por ejemplo) así como de su mundo interior (intenciones, motivaciones y valores, por ejemplo). Tercero, se debe poder dar cuenta de un trabajo reflexivo durante el acto creativo e interpretativo, por fuera de la acción. Y, por último, sus resultados deben conducir a conclusiones bastante generales de manera que pueden llevarse a la teoría, a la práctica creativa o a la enseñanza.

El segundo de los componentes clave de esta definición es la inscripción del conocimiento. Según Clifford Geertz (1994), una inscripción es la fijación de un significado. Es usual que, en 
los procesos de investigación, esta fijación de significados ocurra de forma verbal, ya sea oral o escrita. Sin embargo, los conocimientos que se producen en la práctica creativa son más susceptibles de ser inscritos en una obra, un objeto, una experiencia, un cuerpo, en fin: en una creación (Ballesteros y Beltrán, 2018). En ese sentido, estamos de acuerdo con Macneill (2014) con que el conocimiento que se inscribe en la creación es de carácter práctico, porque es en contacto permanente con los medios y los materiales que se descubren nuevas posibilidades; experiencial, porque los espectadores o usuarios se sumergen en una experiencia estética cuando contemplan una creación; y cognitivo-afectivo, pues dicha creación puede detonar pensamientos y emociones que pueden dar lugar a un conocimiento sobre nosotros mismos, de forma individual o colectiva.

El tercer elemento de esta definición es artefacto plástico-sensorial. De acuerdo con el Diccionario de la Real Academia Española (2014), la palabra artefacto se origina en dos términos latinos: arte factum. Estos quieren decir "hecho con arte". De acuerdo con el mismo diccionario, la palabra arte proviene del latín ars y artis que son calcos del griego téchnē, es decir, técnica en castellano. Técnica y arte tienen el mismo origen; sin embargo, el primero es en griego y el segundo en latín.

Ahora bien: entendemos la técnica en función de las cuatro dimensiones propuestas por Aristóteles: la teoría, que hace referencia a la vida perfecta de la contemplación y del saber abstracto, separado de la vida práctica; la poiesis, que corresponde a la actividad de fabricación o de producción realizada por máquinas (o esclavos y animales); la praxis, que es la acción en el campo moral o de la política; y, finalmente la téchnē que implica la actividad del artesano que puede realizarse por el conocimiento teórico de la naturaleza (von Zuben, 2013).

En ese orden de ideas, proponemos una comprensión de artefacto como algo concretado por el ser humano en un plano material, a partir de un conocimiento abstracto. Siguiendo lo que propone Latour (1987), un hecho (factum), se transforma en artefacto cuando deja de ser abstracto, cuando se inscribe en una entidad material (objeto, texto, cuerpo) y este es reconocido por un grupo social determinado. En ese sentido, Elzen (1986) sostiene que un artefacto se constituye por una "red de aspectos económicos, políticos, culturales, técnicos, 
naturales y sociales" (traducción libre, p. 621) que contiene "elementos heterogéneos que se moldean y se asimilan” (traducción libre, p. 621) a esta, evocando la dimensión de la praxis y de la poiesis.

Siguiendo esa línea de pensamiento, el paso de un hecho — conocimiento abstracto- a un artefacto- inscripción concreta del significado en un ente o ser material- ocurre mediante un testeo continuo de las posibles inscripciones en sistemas como dibujos, bocetos, maquetas, prototipos, sonidos y gestos que permiten entender el comportamiento, los colores, las formas, las texturas, etc. de los materiales y medios (Koskinen, Zimmerman, Binder, Redstrom y Wesveen, 2012). La transición de un sistema a otro mediante las síntesis sucesivas de sus funciones hace emerger distintos significados $y$, poco a poco, se va obteniendo un sistema completamente unificado y coherente consigo mismo (Braudillard, 1968).

Por último, esta integración del sistema se hace mediante la capacidad de manipular, moldear y disponer los distintos elementos perceptuales o plástico-sensoriales, es decir, que se pueden percibir a través de los distintos sentidos por parte del ser humano con el fin de visibilizar ciertas realidades, antes ocultas. Recordemos que para Jacques Rancière (2000), la estética hace alusión a las relaciones que se desprenden de la corporeidad en el campo perceptivo de los seres humanos y, por lo tanto, involucra los condicionamientos de los sentidos que definen nuestro estar y ser en el mundo. Lo sensible, de acuerdo con Arcos Palma (2009), es "el conjunto de realidades que afectan nuestra percepción" (p. 150) y en ese sentido, los creadores tienen la facultad de redistribuir lo sensible a fin de volver perceptibles realidades que antes eran imperceptibles. Esta capacidad se constituye en una potencialidad de producir un nuevo conocimiento que se inscribe en una creación, con una materialidad ${ }^{2}$ de base.

Ante este panorama, estudiantes y profesores de disciplinas creativas deben comprender su función como creadores en procesos de investigación inter y transdisciplinar, con el fin de articularse con otras disciplinas porque la función de los creadores involucra el tránsito del conocimiento teórico o abstracto al concreto, en términos de materialidad o perceptibilidad: deben elaborar los conocimientos de tal suerte que puedan inscribirse en artefactos plástico-

\footnotetext{
${ }^{2}$ Hablamos de "materialidad" como algo que es susceptible de ser percibido por alguno de los sentidos del ser humano.
} 
sensoriales. Findeli et al. (2008) denominan este tránsito — del hecho al artefacto- la transformación del conocimiento y consideran, por lo tanto, que la creación es una actividad transdisciplinar por naturaleza. La transdiciplinariedad busca abordar cuestiones complejas y multidimensionales que, con frecuencia, corresponden a la interfaz entre el ser humano y el mundo (Wickson, Carew y Russell, 2006) y, de hecho, es esta interfaz de la que se ocupa la estética y, por ende, el arte y las demás disciplinas creativas. El propósito último del proceso creativo es la intervención en la habitabilidad del mundo entendida como el acoplamiento entre el cuerpo y los sentidos del ser humano con los distintos estímulos visuales, sonoros, táctiles, olfativos y gustativos que en la corriente continua de la experiencia son percibidos e interpretados. En consecuencia, las formas de intervención que propone la creación implican la manipulación y transformación de las distintas materialidades que parten de la intención de proponer un estímulo sensorial específico que requiere del establecimiento de cómo los seres humanos proyectan y habitan el mundo. Por tanto, la descripción y reflexión de las prácticas sensoriales humanas es fundamental para los procesos creativos y no tienen fronteras disciplinares, precisamente porque la observación fenomenológica busca ocuparse de la experiencia continua e ininterrumpida.

Por un lado, los procesos de creación suelen explorar los principios y las diversas prácticas ontológicas, epistemológicas y metodológicas que les permitan situarse en el contexto que se quiere intervenir, y desarrollar la capacidad de transitar a través de diferentes cuerpos de conocimientos para reflexionar críticamente con relación a ellos, a fin de proponer procesos transformativos de deconstrucción y reconstrucción, que es como describen Wickinson et al. (2006) la transdisciplina. Por otro lado, el momento de crear, es decir, de inscribir un conocimiento abstracto en una materialidad concreta a través de la composición y la configuración, requiere de un proceso de transformación de naturaleza hermenéutica: es decir, el creador debe interpretar o traducir el conocimiento en formas materiales concretas susceptibles de producir estímulos específicos. Este proceso no es automático, sino que consiste en una reelaboración crítica que busca que emerja una nueva realidad a partir de la materialidad propuesta en una unidad integral. Rock y Adler (2019) califican este proceso 
como transdisciplinar en virtud de que diferentes modos de conocimiento se comprometen con un nivel de concreción específico a través de una visión integrativa amplia.

Un ejemplo de la creación como transdisciplina es la integración del arte con la ciencia y la tecnología como lo hace el grupo Atractor Estudio cuya propuesta consiste en videoperformances en las que se combinan la danza, el diseño computacional y la fotografía, "donde el cuerpo de un performer que afecta en tiempo real la modulación del sonido y el video logra generar una narrativa no convencional.” ${ }^{3}$ (Atractor Estudio, 2017, párr. 1). Otro ejemplo de transdisciplina que se puede destacar es la obra de Juan Covelli (2020), quien fusiona la expresión artística con la electrónica, el sonido, la programación, la realidad virtual. ${ }^{4}$ En ambos casos vemos cómo la creación integra e inscribe ciertos tipos de conocimiento experto que se encuentra por fuera de las artes para entregar artefactos sensoriales que buscan expresar un significado concreto.

\section{Fases de la investigación-creación}

Siguiendo la propuesta de Ballesteros y Beltrán (2018), consideramos tres momentos o fases en los procesos creativos: la contextualización, la detonación y la conformación plástica. A continuación, explicaremos en qué consiste cada una de ellas y las iremos ilustrando con el caso del proceso de la obra La Ricarda al final de la pista de la artista española Matilde Obrador (2017), consistente en una propuesta de videoarte.

En primer lugar, la contextualización consiste en el reconocimiento de la realidad en la que surge el proyecto y que se pretende afectar. En esta fase, el creador observa y contempla el entorno de interés desde sus dimensiones políticas, económicas, sociales, culturales, tecnológicas y, por supuesto, estéticas. De acuerdo con García (1989), este es un momento eminentemente cognoscitivo en el que cualquier forma de conocer y apropiar la realidad es bienvenida: la ciencia y el arte se complementan. En palabras de Sadler-Smith (2015), el fenómeno o problema de interés es indagado desde todas las direcciones posibles. Esta fase se caracteriza por el desarrollo de un proceso riguroso, regulado y consciente que ocurren

\footnotetext{
${ }^{3}$ La obra de Atractor Estudio se puede consultar en https://www.atractor.org/copia-de-projects

${ }^{4}$ La obra de Juan Covelli se puede consultar en https://www.juancovelli.xyz/
} 
mediante la activación de la lógica, la documentación y la observación activas. Si se quiere, la contextualización corresponde al momento en el que se formula un proyecto: el investigador creador se dispone a problematizar un fenómeno que le resulte inquietante buscando activamente vacíos en el conocimiento (tanto técnico como conceptual) y posibilidades de propuestas que resulten novedosas. Esto implica la identificación, exploración y apropiación de teorías y conceptos clave que actúen como dispositivos de observación y apropiación de la realidad y del mundo. Por lo tanto, herramientas de investigación de las ciencias naturales y sociales pueden ser de gran utilidad y han de seleccionarse y utilizarse según el objetivo o interés que se persiga.

En la obra La Ricarda al final de la pista (Obrador, 2017), la contextualización ocurre cuando la artista realiza la búsqueda y la recuperación de documentos acerca de una vieja casa ubicada en la ciudad de Barcelona, que alguna vez sirvió de espacio para la música en la década de los años 60, pero que, en la actualidad, es inhabitable por su cercanía al aeropuerto. Enseguida, Obrador visita el lugar en varias ocasiones e inicia un proceso de entrevistas a personas que conocieron dicha morada durante su época dorada. En esta fase de exploración e inmersión en el contexto, la artista no se limita a una mera descripción del espacio, sino que profundiza también en las sensaciones y emociones que se detonan, en una suerte de ejercicio autoetnográfico.

El segundo momento corresponde a la detonación, que entendemos como el proceso cognitivo a través del cual el creador logra comprender los distintos componentes del contexto o del fenómeno de interés detonando una sensación, estrictamente subjetiva, de claridad acerca de cómo puede visibilizar o transformar una realidad que está oculta, «traduciéndola»y «trasladándola» (véase Latour, 1987, p. 117) a una serie de elementos perceptuales y sensoriales. Es cuando la obra está en estado inminente. García (1989), describe esta fase como esencialmente ideológica, dado que el investigador creador establece las relaciones entre los conceptos indagados y las ideas que tiene del mundo, de los otros seres humanos y de la naturaleza, lo que lo puede conducir a asumir una postura política que quiere visibilizar, cuestionar, criticar o interpretar con la obra de arte. En esa fase, ocurre una suerte de “incubación” (traducción libre, Sadler-Smith, 2015, p. 345) caracterizada por la alternancia de 
procesos mentales conscientes e inconscientes y voluntarios e involuntarios en el que se dejan espacios de distracción y relajación, a fin de que el inconsciente pueda encontrar asociaciones y pueda conectar con el preconsciente. En otras palabras: se suscitan zonas liminales de consciencia que se caracterizan por destellos que pueden llegar a durar menos de un segundo y que ocurren en la intimidad del cuerpo-mente del investigador creador quien suele sentir que el destello se le escapa hasta que logra prolongarse en el tiempo y estabilizarse en la consciencia alcanzando un estado de "iluminación” (traducción libre, Sadler-Smith, 2015, p. 346) o consciencia enfocada: ya el investigador creador sabe qué camino seguir. En un proyecto, el proceso de detonación suele ocurrir cuando interpretamos los hallazgos encontrados en campo, que actúan como detonadores, y que nos guían sobre las decisiones a tomar. Dichas decisiones resultan del establecimiento de relaciones entre lo conceptual y lo empírico o experiencial, que no es más que la interpretación.

En el mismo ejemplo de La Ricarda al final de la pista, (Obrador, 2017) la artista busca cómo se puede interpretar el traslape de los relatos que ella construyó en las entrevistas y de sus propias sensaciones y emociones, continuando su proceso autoetnográfico en el que vivencia experiencias cotidianas con alguien que habitó la casa en el pasado. Poco a poco, la obra se iba manifestando en la mente de la artista.

Finalmente, la conformación consiste en la manifestación plástica de la obra. Es decir, es el momento en el que esta adquiere un tipo concreto de materialidad mediante un proceso de exploración que puede empezar con el dibujo o aproximaciones tridimensionales, que se continúa con la técnica propia de la creación y que, por último, concluye con la obra. Retomando a García (1989), esta fase se puede describir como estética, en tanto que se determina la forma de la obra de arte según sus contenidos y consiste en transformar una realidad en otra realidad. El proceso se da en términos de una verificación consciente de los elementos materiales que funcionan y los que no, siguiendo la lógica racional y en función de las estructuras sociales y culturales del contexto (Sadler-Smith, 2015). El momento de conformación plástica es iterativo, es decir, pasa por versiones sucesivas en las que el investigador creador hace énfasis en la diferencia. Siguiendo la propuesta de Ferry (2019), unas nociones de iteración consisten en desempeñar la misma operación múltiples veces usando el 
resultado o salida de la operación anterior como entrada de la siguiente; otras nociones conciben la iteración como diversas expresiones de lo mismo. En los procesos creativos, como en otros procesos culturales descritos por Ferry (2019), la iteración se puede pensar como indicios o signos sucesivos que se enmarcan dentro de circuitos comunicativos y productores de relaciones que van marcando el camino hacia la unidad del artefacto plástico-sensorial que integra el conocimiento producido.

Retomando el caso de la obra La Ricarda al final de la pista (Obrador, 2017), en la fase de conformación plástica, la obra se empieza a manifestar cuando la artista inicia la grabación de las distintas piezas de video; en esta, su intención es darle protagonismo a la cámara como agente expresivo. La conformación continúa en la fase de postproducción, en la que se edita y se ensambla la pieza final cuyo resultado es una obra de videoarte con una duración de 4 minutos y 40 segundos que se presenta en loop, "para enfatizar la emoción en la repetición” (Obrador, 2017, p. 12), en el Festival internacional de videoarte e imagen en movimiento de Barcelona en el año 2014. La obra de videoarte se centra en mostrar a un antiguo visitante de la casa quien recuerda la afinación de los instrumentos, mientras rememora el pasado evocando los sonidos de la música que se van transformando en los ruidos de avión y de las actividades cotidianas que se ven incómodas. "Al final de la pista" hace referencia a que este lugar se le ha acabado la música — la pista musical—para darle paso al sonido de la pista del avión.

Así las cosas, a la hora de formular o informar sobre un proyecto de investigación-creación, se puede empezar describiendo el proceso creativo que va a dar lugar a la creación en términos de estos tres momentos, de manera que se pueda dar cuenta de cómo la práctica creativa puede ser disciplinada y estructurada. Además, este ejercicio obliga a verbalizar lo que se está previendo, proceso en el que se da cuenta de cuáles son los vacíos que se va a tener que llenar. De igual manera, nos da un primer indicio acerca de cómo se puede ir registrando, después, nuestro propio proceso de creación, pues al menos ya tenemos una estructura con la que podemos ir verbalizando las distintas acciones que van dando lugar a la obra y de esta manera, facilitar que su aporte pueda llegar a tener un componente educativo, como lo proponen Findeli y Coste (2007). 


\section{Conclusiones}

En Colombia, aún persiste la dificultad de reconocer que la creación puede producir nuevos conocimientos, dignos de entrar dentro de la categoría de lo que llamamos investigación, a pesar de que grandes filósofos de la ciencia, como Paul Feyerabend (1976) hayan propuesto, desde la década de los años 70, que la producción de nuevos conocimientos esté más cerca de los procesos de creación que de la aplicación de un método que pretendió ser inequívoco y universal. Por su parte, filósofos del arte como Jacques Rancière (2000) o HansGeorg Gadamer (1996) también han propuesto que la experiencia estética produce conocimientos que no pueden accederse sino a través del arte y que distan considerablemente del conocimiento que se produce desde los procedimientos científicos, los cuales resultan reduccionistas. En ese sentido, la hegemonía del positivismo hace mella en los sistemas nacionales de investigación, en particular en aquellos del sur global, que terminan intentando adaptarse a las normas impuestas por sistemas pensados para otras latitudes y desconociendo la potencialidad que tiene la investigación-creación como la posibilidad de darle voz a nuestras diferencias en contraposición con lo universal.

Además, aún nos pesa la herencia de la separación milenaria conceptual entre ciencia y arte que no viene siendo otra cara de la dualidad entre teoría y técnica. Consideramos, sin embargo, que estas dos facetas de la realidad constituyen un continuo. De hecho, varias de las referencias que utilizamos en la argumentación presentada a lo largo de este texto provienen de los estudios sociales de la ciencia. Más allá de las iniciativas interdisciplinares y transdisciplinares, acoger la otredad se constituye en un descubrimiento de nuevas maneras de pensar, de ver el mundo y de construir nuevas realidades más incluyentes. Desafortunadamente, el modelo dominante de conocimiento ha correspondido a las ciencias y se ha basado en la reproducibilidad del método y generalización de los resultados porque este ha permitido la manipulación, gestión y control de los fenómenos naturales. Esto ha excluido cualquier otro modo de producción de conocimiento planteado por las experiencias extracientíficas que no necesariamente buscan controlar. El arte nos permite acceder a experiencias de conocimiento, que, aunque singulares y particulares, nos abren a visibilizar realidades y nuevos mundos. 
Finalmente, este artículo buscó proponer una estructura argumentativa, en términos de las características del conocimiento que produce la investigación-creación y de sus momentos o fases, que le sea útil a los creadores a la hora de justificar cómo los procesos de creación, en ocasiones, pueden calificarse de investigativos en tanto que producen nuevos conocimientos, cuya característica principal es que se inscriben en artefactos plástico-sensoriales. La función del creador es transformar estos conocimientos en elementos perceptuales específicos que aporten significativamente al contexto desde el punto de vista estético para volver visible lo invisible, invitándonos a pensarnos como sociedad y como humanidad. He ahí el nuevo conocimiento que se produce en las artes y el importante valor que tiene.

\section{Referencias}

Aguilar, B., Mahecha, I. (2011). Cuerpo femenino y abyección en los desnudos de Débora Arango. Cuadernos Kóre. Revista de historia y pensamiento de género, 1(5), 121-15.

Arcos Palma, R. J. (2009). La estética y su dimensión política según Jacques Rancière. Nómadas, (31), 137-155.

Asprilla, L. I. (2014). Evaluación de los productos de la creación-investigación. La producción de conocimiento desde las artes. Bogotá: Acofartes.

Artefacto. (2014) En Diccionario de la Lengua española (23a ed.). Recuperado de https://dle.rae.es/artefacto?m=form

Arte. (2014) En Diccionario de la Lengua española (23a ed.). Recuperado de $\underline{\text { https://dle.rae.es/arte? } \mathrm{m}=\text { form }}$

Atractor Estudio. (2107). Atractor estudio. Recuperado de https://www.atractor.org/inicioes

Ballesteros, M., \& Beltrán, E. M. (2018). ¿Investigar creando? Una guia para la investigacióncreación en la academia. Bogotá: Universidad El Bosque. 
Beltrán, E. M. (en prensa). Dificultades y resistencias en la investigación-creación: una propuesta de estructura y método. Ponencia presentada en el Seminario Internacional Arte \& Alteridad.

Baudrillard, J. (1968). Le système des objets. Paris: Gallimard.

Borgdorff, H. (2007). The debate on research in the arts. Dutch Journal of Music Theory, 12(1), 1-17.

Covelli, J. (2020) Juan Covelli. Recuperado de https://www.juancovelli.xyz/

Departamento Administrativo de Ciencia, Tecnología e Innovación, Colciencias. (2016). Participación y aportes de la Comunidad de Artes, Arquitectura y Diseño en el Modelo en el Reconocimiento y Medición de Grupos de Investigación, Desarrollo Tecnológico o de Innovación y en el Reconocimiento de Investigadores del Sistema Nacional de Ciencia, Tecnología e Innovación. Bogotá: Colciencias.

Elzen, B. (1986). Two ultracentrifuges: A comparative study of the social construction of artefacts. Social Studies of Science, 16(4), 621-662.

Ferry, E. (20 de diciembre de 2019). Iterations. Recuperado de https://culanth.org/fieldsights/iterations

Feyerabend, P. K. (1976). Contra el método. Esquema de una teoría anarquista del conocimiento (F. Hernán, Trad.). Barcelona: Ariel Quincenal.

Findeli, A. (1998). Will design ever become a science? Epistemological and methodological issues in design research, followed by a preposition. En P. Strandman (Ed.) No Guru, no Method? (pp. 63-69). Helsinki: University of Art and Design Helsinki.

Findeli, A., \& Coste, A. (2007). De la recherche-création à la recherche-projet: Un cadre théorique et méthodologique pour la recherche architecturale. Lieux Communs, (10), 139-161. 
Findeli, A., Brouillet, D., Martin, S., Moineau, C., \& Tarrago, R. (2008). Research through design and transdisciplinarity: A tentative contribution to the methodology of design research. Paper presented at the "Focused"-Current Design Research Projects and Methods, 67-91.

Frayling, C. (1993). Research in Art and Design. Royal College of Arts Research Papers, 1(1), 15.

Gadamer, H. (1996). Verdad y método I (A. A. Aparicio, R. de Agapito, Trads.). (6ta ed.). Salamanca: Ediciones Sígueme.

García, S. (1989). Teoría y práctica del teatro (2 $2^{\mathrm{da}}$ ed.). Bogotá: Ediciones La Candelaria.

Geertz, C. (1994). Conocimiento local (A. López Bargados, Trad.). Barcelona: Paidós.

Hannula, M., Suoranta, J., \& Vaden, T. (2005). Artistic research. Theories, methods and practices. Helsinki \& Gothemburg: Academy of Fine Arts.

Hatch, A. (2019). To fix research assessment, swap slogans for definitions. Nature, 576, 9.

Koskinen, I., Zimmerman, J., Binder, T., Redström, J. \& Wensveen, S. (2012). Design Research through Practice. Waltham: Elsevier.

Latour, B. (1987). Science in action. Cambridge, Massachusetts: Harvard University Press.

Londoño, F. C. (2013). Enfoques de la investigación-creación en programas de arte y diseño. En M.V. Casas Figueroa (Ed.). Memorias del evento de valoración de los procesos de creación artística y cultural en el marco de la acreditación de programas (pp. 67-76). Bogotá: Ministerio de Educación Nacional.

Macneill, P. (2014). Ethics and the arts: A critical review of the new moralisms. En P. Macneill (Ed.), Ethics and the arts (pp. 167-177). Nueva York- Londres: Springer. 
Martínez, J. (2020) Un territorio acechado: realismo espectral en Fragmentos de Doris Salcedo. Revista de estudios colombianos, (55), 42-49.

NC-Arte. (2014). NC-LAB, Laboratorio de pensamiento creativo. Memorias 2014. Bogotá:

Fundación Neme. Recuperado de http://www.nc-arte.org/wpcontent/uploads/2015/06/Memorias-NCLAB2014-0415.pdf

Obrador, M. (2017). Memoria del videoarte La Ricarda al final de la pista. Génesis de una narrativa digital. Barcelona: Editorial DigiDoc.

Rancière, J. (2000). Le partage du sensible. París: La Fabrique.

Rock, J., \& Adler, S. (2019). From 'SciArt' to 'Art science'. Antennae, (47), 14-25.

Niedderer, K. (2013). Explorative materiality and knowledge. The role of creative exploration and artefacts in design research. FORMakademisk, 6(2), 1-20.

Salcedo, D. (2018) Fragmentos. Bogotá: Carrera 7B N 6b -30.

Sadler-Smith, E. (2015). Wallas' four-stage model of the creative process:

More than meets the eye? Creativity Research Journal, 27(4), 342-352.

doi:10.1080/10400419.2015.1087277

Scrivener, S. (2002). The art object does not embody a form of knowledge. Working Papers in Art and Design 2. Recuperado de https://www.herts.ac.uk/_data/assets/pdf_file/0008/12311/WPIAAD_vol2_scrive ner.pdf

von Zuben, N. A. (2013). Bioética: Nuevas tiranías y fundamentalismos. En L. Pessini, J. E. de Siqueira \& W. S. Hossne (Eds.), Bioética en tiempos de incertidumbre (pp. 205226). Bogotá: Universidad El Bosque. 
Wickson, F., Carew, A. L., \& Russell, A. W. (2006). Transdisciplinary research: Characteristics, quandaries and quality. Futures, 38(9), 1046-1059. Doi 10.1016/j.futures.2006.02.011

Enviado: 2020-03-16

Aceptado: 2020-12-07 